\title{
Síndrome de dehiscencia del canal semicircular superior. A propósito de un caso
}

\section{Canal dehiscence syndrome of the superior semicircular. In a case purpose}

Víctor Mercado $\mathbf{M}^{1}$, Francisca Fernández $\mathrm{A}^{2}$, Claudia Hernández $\mathrm{B}^{3}$, Carlos Pino $\mathrm{U}^{3}$, Ignacio Novoa $\mathrm{C}^{4}$, Pablo Herrera $\mathbf{C}^{5}$.

\section{RESUMEN}

El síndrome de dehiscencia de canal semicircular superior (DCSS) es una patología descrita en 1998 por Minor y cols, presenta síntomas diversos incluido vértigo inducido por el sonido, hipoacusia y autofonía por la falta de cobertura ósea en dicho canal. El diagnóstico se basa en la clínica y la confirmación se obtiene mediante la tomografía computarizada de peñasco. El tratamiento será expectante o reparación quirúrgica de la continuidad si la clínica es incapacitante. En este artículo se presenta un caso clínico de DCSS con síntomas auditivos y vestibulares al emitir el fonema " $m m m$ ".

Palabras clave: Canal semicircular superior (CSS), dehiscencia, fenómeno de TuIlio.

\begin{abstract}
The superior semicircular dehiscence sindrome is a pathology described en 1998 by Minor et al. Which presents several symptoms incluid sound induced vértigo, hearing loss and autophony due to bone dehiscense of this semicircular canal. The diagnosis was based on clinical and confirmation is given by the temporal bone CT. Treatment is expectant or surgical repair of continuity if the clinic is disabling. In this paper we present a case of DCSS with auditory and vestibular symptoms in issuing the phoneme "mmm".
\end{abstract}

Key words: Superior semicircular canal, Dehiscence, Tullio phenomenon.

\footnotetext{
Médico Otorrinolaringólogo, Universidad de Valparaíso.

2 Residente Otorrinolaringología, Hospital Carlos van Buren, Universidad del Valparaíso.

3 Tecnólogo Médico, Marina Médica, Viña del Mar.

4 Licenciado en Kinesiología, Universidad Andrés Bello, Viña del Mar.

5 Médico Radiólogo, Clínica Reñaca, Viña del Mar.
}

Recibido el 24 de agosto de 2015. Aceptado el 18 de septiembre de 2015. 


\section{INTRODUCCIÓN}

El síndrome de DCSS consiste en la presencia de una solución de continuidad entre el ápex del canal semicircular superior (CSS) y la fosa cerebral media' . Minor y cols fueron los primeros en identificar este síndrome en 1998, y se caracteriza por la presencia de vértigo y nistagmo frente a sonidos, cambios de presión en el oído medio y/o presión intracraneal. Además pueden aparecer síntomas como autofonía, inestabilidad, oscilopsia e hiperacusia. Minor planteó que la falla de cobertura ósea o dehiscencia actuaría como una "tercera ventana" móvil a nivel del oído interno en forma adicional a las ventanas oval y redonda, lo que permitiría la transmisión de la vibración hacia el aparato vestibular, produciendo la sensación de vértigo². El mecanismo involucrado en el síndrome de DCSS sería el movimiento de la ampolla del canal debido a una complacencia aumentada del sistema endolinfático, generada por la dehiscencia ${ }^{3}$.

Un estudio de prevalencia ${ }^{4}$ estudiando mil huesos temporales se apreció una DCSS en el 0,5\% de Ios casos, la lámina ósea que recubría el CSS era menor de $0,1 \mathrm{~mm}^{2}$ en el $1,4 \%$. No se encontraron alteraciones locales en el hueso temporal que pudieran explicar la erosión ósea, y ninguno de estos pacientes tenía historia previa de traumatismo craneal. Esta dehiscencia era bilateral en la mayoría de los pacientes, por lo que se plantea que alguna alteración embrionaria podría explicar estos hallazgos, y que, probablemente algún evento posterior, como un traumatismo 0 aumento de la presión intracraneana, podría acentuar esta condición, determinando la aparición de síntomas 4 .

Experimentos iniciales realizados por Tullio ${ }^{5} \mathrm{y}$ posteriormente por Huizinga ${ }^{6}$ y Euren ${ }^{7}$ demostraron que la fenestración de canales semicirculares en palomas, provocaba movimientos oculares y cefálicos evocados por sonido en el mismo plano del canal, denominándose fenómeno de Tullio. Estudios clínicos iniciales identificaron este fenómeno en pacientes con sífilis congénita ${ }^{8}$. Sin embargo, estos hallazgos fueron posteriormente demostrados en patologías como sordera congénita9, síndrome de Ménière ${ }^{10}$, fístula perilinfática ${ }^{11}$, traumatismo encefalocraneano ${ }^{12}$, enfermedad de Lyme $^{13}$ y otitis media crónica colesteatomatosa con erosión del canal semicircular ${ }^{14}$.
En estados patológicos similares a los que puede resultar el fenómeno de Tullio, la presión en el conducto auditivo externo (CAE) con el consiguiente movimiento de la membrana timpánica, produciría síntomas y signos vestibulares ${ }^{15}$. Este fenómeno fue demostrado en un estudio realizado con chinchillas por Hirvonen y cols, quienes investigaron los cambios de descarga en fibras aferentes vestibulares en respuesta a cambios en el CAE antes y después de la fenestración del CSS, y posterior a la reparación ${ }^{16}$. Esta relación entre el plano y las características direccionales de los movimientos oculares evocados conocidos tanto por la excitación o inhibición del CSS ayudarán a definir las características fisiopatológicas de los pacientes. Aparecerá entonces, un nistagmo vértico-rotatorio siguiendo el plano del CSS afectado, en sentido antihorario en el caso del CSS derecho y en sentido horario en el CSS izquierdo ${ }^{17}$. La confirmación mediante la tomografía computarizada (TC) de peñasco de alta resolución se deberá realizar con cortes de al menos $0,5 \mathrm{~mm}$ y reconstrucción en el plano del canal para minimizar el número de falsos positivos ${ }^{18}$. Otro examen complementario de utilidad para el diagnóstico de este cuadro son los potenciales evocados miogénicos vestibulares (VEMP). Observándose un aumento en la amplitud y un menor umbral para evocar los potenciales ${ }^{19}$. Halmagyi y cols evaluaron los movimientos oculares en respuesta a clicks de baja frecuencia en pacientes portadores de DCSS y grupo control sano. El grupo portador tenía una respuesta diez veces mayor a la observada en sujetos sanos, por lo que plantean esta técnica como una alternativa válida de pesquisa para DCSS en pacientes con vértigo y/0 desequilibrio ${ }^{20}$.

\section{CASO CLÍNICO}

Paciente sexo masculino, 33 años de edad, administrativo en minería. Consulta por cuadro de dos años de evolución caracterizado por hipoacusia, autofonía y tinitus pulsátil del oído izquierdo. En los últimos ocho meses se agrega desequilibrio y vértigo objetivo al emitir el fonema "mmm". Al examen destaca otoscopia normal; test de Weber lateralizado a oído izquierdo. La audiometría, presenta hipoacusia de conducción en 
oído izquierdo de curva ascendente y un Gap de 40 dB (125-250-500 Hz), vía ósea en -10dB (Figura 1). Con la sospecha clínica y audiológica de una DCSS se solicita VEMP con estímulo Burst de $500 \mathrm{~Hz}$, que presenta un umbral de las ondas p13 y n23 a $65 \mathrm{~dB}$ $\mathrm{nHL}$ y asimetría en las amplitudes de las ondas $\mathrm{p} 13$ y n23 a $90 \mathrm{~dB}$ nHI (Figura 2).
La video-oculonistagmografía presenta ausencia de nistagmo espontáneo. Se solicita al paciente realizar fonema "mmm" observándose aparición de nistagmo horizonto-rotatorio en todas las posiciones de la mirada, en sentido horario (Figura 3). A la prueba calórica se observa una hiperreflexia vestibular izquierda significativa según parámetro

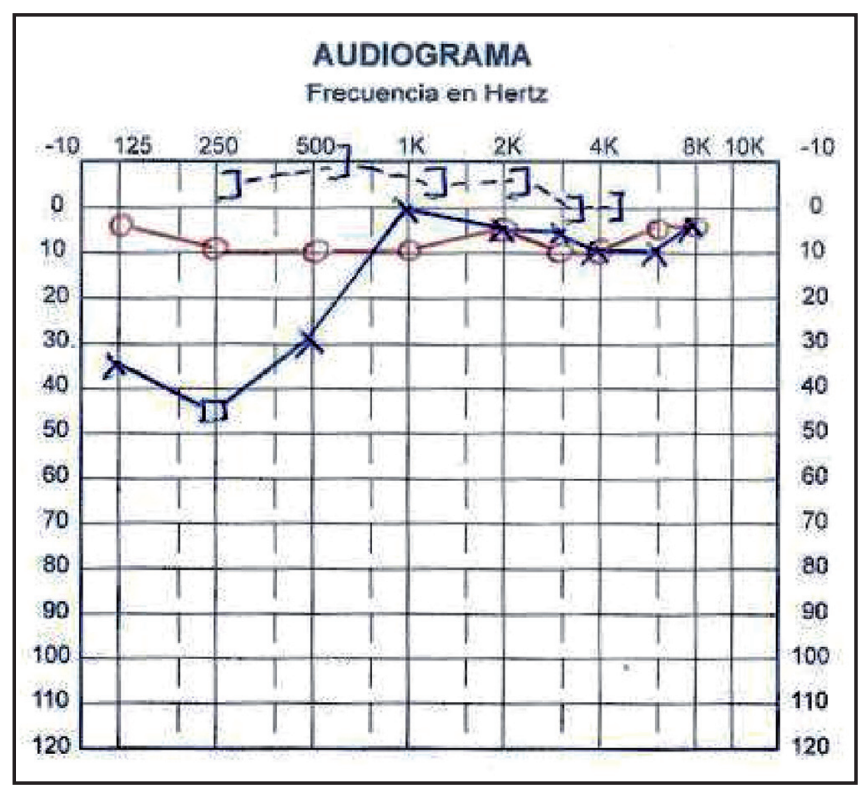

Figura 1. Hipoacusia conductiva de oído izquierdo, ascendente. Y GAP de 40 dB. , vía ósea -10 dB.

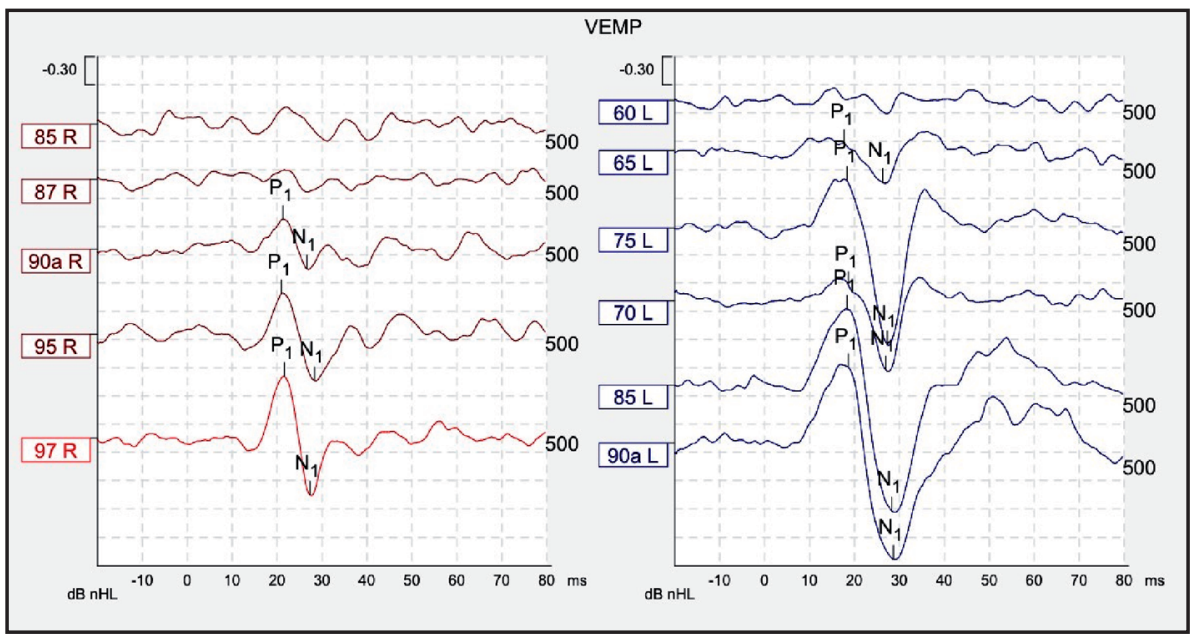

Figura 2. Asimetría en la amplitud de las respuestas, umbral de respuesta anormalmente reducido en oído izquierdo. Compatible con patología vestibular izquierda. 


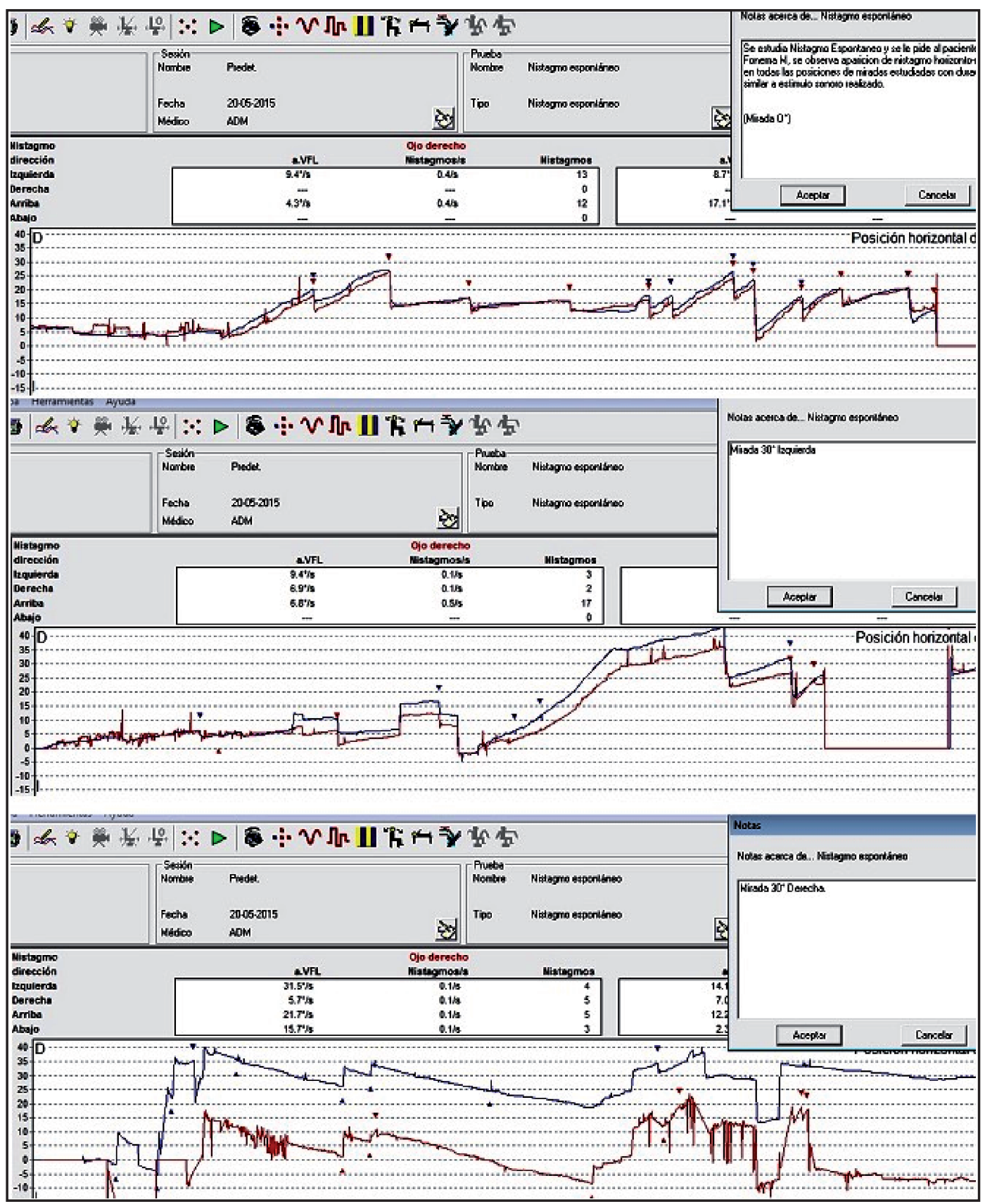

Figura 3. Se estudia nistagmo espontáneo, y se pide al paciente emitir fonema "mmm", se observa aparición de nistagmo horizonto-rotatorio a izquierda en todas las posiciones de la mirada.

Descripción de la adquisición: Se realizó en equipo Toshiba Aquilion 64, Yokohama, Japón. Adquisición de 0,5 mm x 64. 120 Kv. 300 mAs. Matriz de 512 x 512. FOV de 240 mm. Reconstrucción de $1 \mathrm{~mm}$ cada 0,8 mm con algoritmo Bone. Tiempo de rotación de 0,6 seg. 


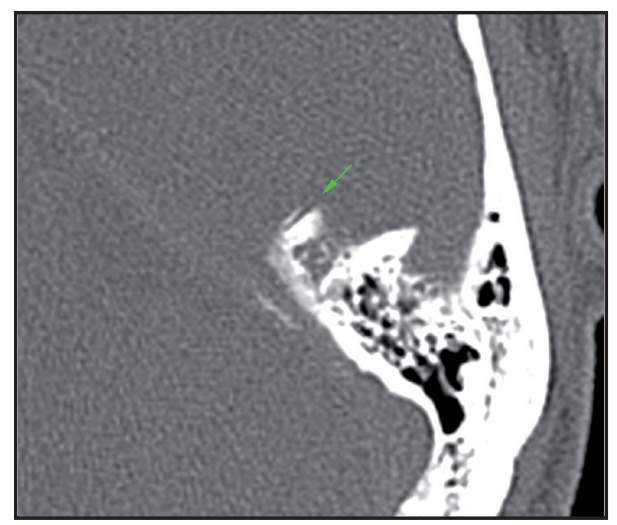

Figura 4a. Reconstrucciónn MPR oblicuo paralelo al plano del tegmen timpánico. Nivel de ventana de $600 \mathrm{UH}$ y ancho de ventana de $2.500 \mathrm{UH}$. La flecha verde señala el CSCS que carece de su pared ósea superior.

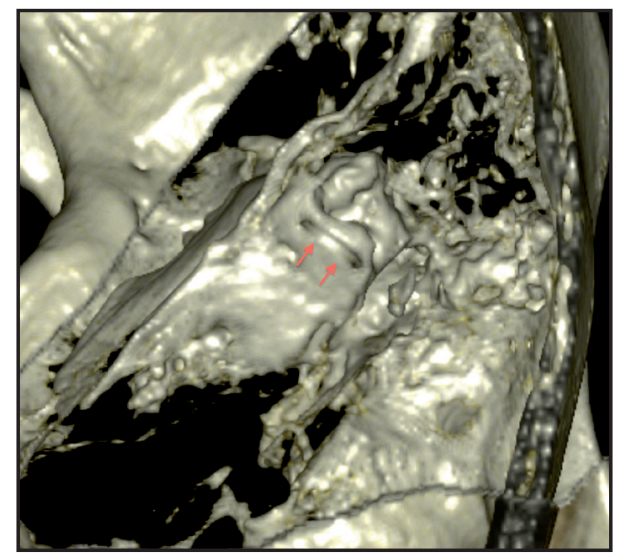

Figura 4b. Reconstrucción con volumen Rendering destacando la superficie ósea adyacente al defecto de la pared superior del CSCS, mostrado con flechas rojas.

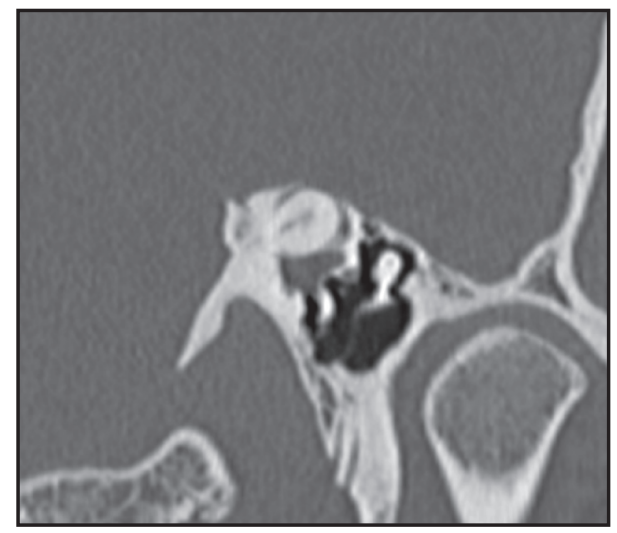

Figura 4c. MPR oblicuo paralelo al plano del CSCS donde destaca la dehiscencia de la pared ósea.
VCL. Con la sospecha clínica de síndrome de DCSS en oído izquierdo se realiza TC de hueso temporal, con cortes de $0,5 \mathrm{~mm}$, que confirma el diagnóstico clínico. (Figuras 4a, 4b, 4c).

\section{CONCLUSIÓN}

La DCSS es una entidad relativamente nueva. La mayoría de las publicaciones dan pocos detalles de la sintomatología, mencionando principalmente desequilibrio, vértigo u oscilopsia inducida por ruido 0 maniobras de presión. En una serie reportada por Kaski ${ }^{21}$, informa que $89 \%$ de los pacientes presentaban desequilibrio, oscilopsia 0 vértigo ante la presencia de sonidos externos intensos de aparición súbita, como el teléfono, ruido de tráfico, llanto de bebe, chillido o fresa dental, ampliando la forma de presentación clínica de una DCSS. Un tercio de ellos presentaban fenómeno de Tullio con su propia voz al hablar 0 al hacer zumbido. Los cambios en la presión intracraneal al toser, sonarse la nariz, viajar en avión, causaban síntomas en el $25 \%$ de los sujetos. Además, los pacientes a menudo informaban fenómenos auditivos inusuales relacionados con sonidos corporales, como oír sus pasos, sus músculos al masticar o los movimientos de los ojos. El tinitus pulsátil fue una queja frecuente. Es importante considerar el vértigo que se presenta en la consulta odontológica, y realizar el diagnostico diferencial entre el vértigo posicional paroxístico benigno (VPPB) y DCSS, ya que se desencadenarán de manera diferente. La intensidad del sonido generado por la turbina alcanza el oído interno por vía ósea, éste se transmite por medios sólidos, como es el cráneo, con enorme eficacia y máxima velocidad. Sin embargo, cuando éste alcanza el oído de un paciente con DCSS se produce un fenómeno de Tullio, pues supera los $95 \mathrm{~dB}$ SPL precisos para desencadenar un vértigo rotatorio. Todas estas molestias mencionadas previamente son provocadas con sonidos de baja frecuencia $(0,5-2 \mathrm{kHz})$. El paciente reportado, en particular refería síntomas al emitir el fonema "mmm", el que genera cambios de presión, así como una resonancia retrorrefleja, porque se ocluye parcialmente el tubo fonatorio, con una frecuencia aproximada de $110 \mathrm{~Hz}$, pero incluye armónicos y otras frecuencias complejas con 
ruido. Dada la naturaleza del problema, se deben buscar movimientos oculares anormales frente a la exposición a estímulos sonoros, inducción de presión positiva y/o negativa en el CAE durante la impedanciometría o la realización de maniobra de Valsalva contra glotis abierta y glotis cerrada. El aumento de la presión intracraneana con la maniobra de Valsalva y glotis cerrada, es transmitida en el CSS, a través de las meninges y la perilinfa del acueducto coclear ${ }^{21}$, generando movimientos oculares en el mismo plano pero de dirección opuesta (ampulípeta) a la observada durante el ruido fuerte. Fisiopatológicamente la "tercera ventana" provoca una hipoacusia conductiva para frecuencias bajas que va acompañada de umbrales de vía ósea normales 0 incluso menores a $0 \mathrm{~dB}$, con un reflejo estapedial normal, lo que nos permitirá excluir la otoesclerosis como diagnóstico diferencial. La conducción ósea de los seres humanos es menos eficiente que la conducción aérea, sin embargo, la presencia de una DCSS generará una vibración del cráneo que podría causar la oscilación de la duramadre sobre el defecto, llevando a la creación de ondas estacionarias y amplificación del sonido, el que se transmite directamente a los fluidos del oído interno con poca atenuación, causando estimulación de las células ciliadas de la cóclea en las frecuencias apropiadas. Esto explica los síntomas de autofonía y tinitus pulsátil22.

EI VEMP es una prueba objetiva que evalúa la generación del reflejo disináptico vestíbulo-cervical. Éste determina el funcionamiento del sáculo, laberinto posterior y nervio vestibular inferior principalmente ${ }^{23-24}$. Se piensa que el VEMP se origina por estimulación del sáculo debido a que este último constituye el órgano terminal vestibular más sensible al sonido, al estar cercano a la ventana oval. La gran intensidad del movimiento de la perilinfa producirá una mayor estimulación de los receptores saculares. Por otro lado, la mayoría de las neuronas del nervio vestibular sensibles a clicks, que además responden a inclinaciones, se encuentran en la mácula sacular, y de ahí se dirigen por las fibras aferentes del nervio vestibular al núcleo vestibular lateral e inferior ${ }^{25}$. De esta manera, un estímulo auditivo breve tipo click de alta intensidad, producirá un potencial inhibitorio ipsilateral de gran amplitud y breve latencia en el músculo esternocleidomastoideo cuando éste se encuentre contraído en forma tónica, y excitatorio en el músculo contralateral. Esta respuesta muscular será representada como una onda con un peak positivo a los 13 ms después y un peak negativo a los 23 ms después de la estimulación. En sujetos normales el VEMP tiene un umbral habitualmente de 90-95 dB. En pacientes con DCSS, el umbral es de unos $20 \mathrm{~dB}$ inferior a los sujetos normales y la amplitud al nivel habitual de estimulación de 100-105 dB puede ser anormalmente alta $(>300 \mu \mathrm{V})$. Los pacientes con DCSS tienen también un reflejo vestíbulo oculomotor evocado por click anormalmente intenso y de umbral bajo $0^{26-27}$.

Existe una notable diferencia entre la incidencia radiológica y anatómica. Mientras en estudios post morten en huesos temporales se encontró una incidencia del $0,5 \%{ }^{4}$, lo que contrasta con los resultados obtenidos mediante TC, que varían desde el $1 \% 28$ al $17 \%{ }^{29}$. Esto puede deberse a que la resolución de la TC no sea suficiente para delinear hueso muy fino $0^{30}$; la orientación de los cortes puede aumentar el número de falsos positivos ${ }^{31} \mathrm{y}$ el posicionamiento de la cabeza del paciente varía con cada estudio y por consiguiente no existe una variable estandarizada.

Cloutier ${ }^{32}$ propuso la realización de TC con reconstrucción en el plano del canal comprometido (Pöschl), reduciendo la prevalencia radiológica de DCSS del $10 \%$ al $4 \%$ con reconstrucciones coronales. No obstante aún se realiza un sobre diagnóstico de este cuadro.

El tratamiento inicial de esta patología debe ser conservador, evitando por parte del paciente, las acciones que desencadenen la clínica². Si esta conducta no es suficiente o los síntomas son incapacitantes se podrá plantear al paciente una resolución quirúrgica ${ }^{3}$, que consistirá en el cierre de la comunicación entre el canal y el suelo de la fosa media, observándose una franca mejoría de la sintomatología vestibular y desaparición del fenómeno de Tullio. Las vías propuestas para el cierre de la fístula son transmastoidea 0 a través de fosa craneal media. Una vez identificada la dehiscencia, ésta es sellada con cera de hueso o un injerto óseo pudiéndose añadir fascia de músculo temporal ${ }^{33}$. Otras alternativas como fascia, goma de fibrina, injerto óseo y cemento de hidroxiapatita han sido utilizados también en la reparación del defecto, con 
diversos resultados ${ }^{1}$. Existe un enfoque innovador descrito inicialmente por Silverstein ${ }^{34}$, quien sugirió que amortiguar la sensibilidad del oído interno mediante el refuerzo de la ventana oval y redonda con fascia podría aliviar los síntomas en algunos pacientes, reduciendo las complicaciones de aquellos abordajes más invasivos, por vía transcraneal. Se estudiaron veintidós pacientes de cuatro centros que optaron por el abordaje mínimamente invasivo mediante el refuerzo de la ventana oval y/o redonda. El análisis reveló una mejoría estadísticamente significativa en casi todos los síntomas a excepción de la pérdida auditiva.

Para finalizar, esta entidad clínica tiene una alta tasa de falsos positivos y una gran variedad de formas clínicas de presentación por consiguiente debemos considerar detalladamente los diagnósticos diferenciales y con el apoyo de los exámenes complementarios llegar plantear este diagnóstico con certeza y definir el mejor tratamiento para aquellos paciente portadores de DCSS.

\section{BIBLIOGRAFÍA}

1. Gianoli GJ. Deficiency of the superior semicircular canal. Curr Opin Otolaryngol Head and Neck Surg 2001; 9: 336-341.

2. Minor LB, Solomon D, Zinreich JS, Zee DS. Soundand/or pressure- induced vértigo due to bone dehiscence of the superior semicircular canal. Arch Otolaryngol Head Neck Surg 1998; 124: 249-58.

3. Caro J, Fernández F. Síndrome de dehiscencia del canal semicircular superior: Revisión. Rev Otorrinolaringol Cir Cabeza Cuello 2006; 66: 119-25.

4. Carey JR, Minor lB, Nager GT. Dehiscence and thinning of bone overlying the superior semicircular canal in a temporal bone survey. Arch Otolaryngol Head Neck Surg 2000; 126: 137-47.

5. Tullio P Das. Ohr und die Entstehung der Sprache und Schrift. Berlin, Germany Urban \& Schwarzenberg 1929.

6. Huizinga E. On the sound reactions of Tullio. Acta Otolaryngol (Stockh) 1935; 22: 359-69.

7. Eunen AJH, Huizinga HC, Huizinga E, Die Tulliosche. Reaktion in Zusammenhang mit der Funktion des
Mittelohres. Acta Otolaryngol (Stockh) 1943; 31 : 265-339.

8. Mayer Fraser JS. Pathological changes in the ear in late congenital syphilis. J Laryngol Otol 1936; 51: 683-714.

9. KWEE SL.The occurrence of the Tullio phenomenon in congenitally deaf children. $J$ Laryngol Otol 1976; 90: 501-7.

10. Kacker SK, Hincholiffe R. Unusual Tullio phenomena. J Laryngol Otol 1970; 84: 15566.

11. Fox EJ, Balkany TJ, Arenberg IK. The Tullio phenomenon and perilymph fistula. Otolaryngol Head Neck Surg 1988; 98: 88-9.

12. Rottach KG, von Maydell RD, DiScenna AO, Zivotofsky AZ, Averbuch-Heller AZ, Leigh RJ. Quantitative measurements of eye movements in a patient with Tullio phenomenon. $J$ Vestib Res 1996; 6: 255-9.

13. Nields JA Kveton JF. Tullio phenomenon and seronegative Lyme borreliosis. Lancet 1991; 338: 128-9.

14. Ishizaki H, Pyykko I, Aalto H, Starck J. Tullio phenomenon and postural stability: experimental study in normal subjects and patients with vertigo. Ann Otol Rhinol Laryngol 1991; 100: 976-83.

15. Hennebert C. A new syndrome in hereditary syphilis of the labyrinth. Presse Med Belg Brux 1911; 63: 467.

16. Hirvonen TP, Carey JP, Liang CJ, Minor LB. Superior canal dehiscence: Mechanisms of pressure sensitivity in a chinchilla model. Arch Otolaryngol Head Neck Surg 2001; 127: 1331-6.

17. Sung KB, Lee TK, Furman JM. Abnormal eye movements in dizzy patients. Neurol Clin 2005; 23: 675-703.

18. Williamson Ra, Vrabec JT, Coker NJ, Sanduin M. Coronal scan prevalence of superior semicircular canal dehiscence. Otolaryngol Head Neck Surg 2003; 129: 481-9.

19. Welgampola MS, Colebatch JG. Characteristics and clinical application of vestibular-evoked myogenic potencials. Neurology 2005; 64: 1682-8.

20. Halmagy GM, McGarvie LA, Yavor RA, Todd MJ. The clic evoked vestíbulo-ocular reflex in superior semicircular canal dehiscence. Neurology 2003; 60: 1172-5. 
21. Kasky D, DaVies R, Luxon L. The Tullio phenomenon: a neurologically neglected presentation. J Neurol 2012; 259: 4-21.

22. Tilikete C, Krolak-Salmon P, Truy E, Vighetto A. Pulse synchronous eye oscillations revealing bone superior canal dehiscence. Ann Neurol 2004; 56: 556-60.

23. Colebatch JG, Halmagyi GM, Skuse NF. Myogenic potentials generated by click-evoked vestibulocollic reflex. J Neurol Neurosurg Psychiatry 1994; 57: 190-7.

24. Watson SR, Halmagy GM, Colebatch JG. Vestibular hypersensivity to sound (Tullio phenomenon): structural and functional assessment. Neurology 2000; 54: 722-8.

25. Halmagy GM, Curtíos IS. Pruebas de función otolítica. Rev Med Univ Navarra 2003; 47: 2937.

26. Halmagyı GM, McGarvie LA, Aw ST, Yavor RA, Todd MJ. Theclickevokedvestibulo-ocular reflex in superior semicircular canal dehiscence. Neurology 2003; 60: 1172-5.

27. Halmagy GM, Aw ST, McGarvie LA, Todd MJ, Bradshaw A, Yavor RA, Fagan PA. Superior semicircular canal dehiscence simulating otosclerosis. J Laryngol Otol 2003; 117: 553-7.

28. Krombach GA, Di Martino E, Schmitz- Rode T, Prescher A and cols. Posterior semicircular canal dehiscence: a morphologic cause of vertigo similar to superior semicircular canal dehiscence. Eur Radiol 2003; 13: 1444-50.

29. Piton J, Negrevergne M, Portmann D. Dehiscence of the superior semicircular canal: approach and CT scan classifications. Rev Laryngol Otol Rhinol 2008; 129: 17-26.

30. Whyte J, Martínez C, Cisneros A. Dehiscencia del canal semicircular superior: Estudio de la incidencia anatómica. Rev Otorrinolaringol Cir Cabeza Cuello 2011; 71: 39-43.

31. Belden CJ, Weg N, Minor LB. Zinreich SJ. CT evaluation of bone dehiscence of the superior semicircular canal as a cause of sound and/or pressure-induced vertigo. Radiology 2003; 226: 337-43.

32. Cloutier JF, Belair M, Saliba I. Superior semicircular canal dehiscence: positive predictive value of high-resolution CT scanning. Eur Arch Otorhinolaryngol 2008; 265(12): 1455-60.

33. Crovetto de la Torre MA, Whyte Orozco J, Cisneros Gimeno Al, Basurko Aboitz , Oleaga Zufiria L, Sarrat ToRREguitart R. Síndrome de dehiscencia del canal semicircular superior. Aspectos embriológicos y quirúrgicos. Acta Otorrinolaringol Esp 2005; 56 : 6-11.

34. Silverstein H, Kartush JM, Parnes LS, Poe DS, Babu SC. LeVEnson MJ. Round window reinforcement for superior semicircular canal dehiscence: A retrospective multi-center case series. Am J Otolaryngol 2014; 35(3): 286-93. 